\title{
AN ARITHMETIC Operation ON HEXADECAGONAL FUZZY NUMBER
}

\author{
Dr.A.Sahaya Sudha ${ }^{1}$ and R .Gokilamani ${ }^{2}$ \\ ${ }^{1}$ Department of Mathematics, Nirmala College for Women, Coimbatore \\ ${ }^{2}$ Department of Mathematics, Sri Ramakrishna College of Arts and Science for Women, \\ Coimbatore
}

\begin{abstract}
In this paper, a new form of fuzzy number named as Hexadecagonal Fuzzy Number is introduced as it is not possible to restrict the membership function to any specific form. The $\alpha-$ cut of Hexadecagonal fuzzy number is defined and basic arithmetic operations are performed using interval arithmetic of $\alpha-c u t$ and illustrated with numerical examples.
\end{abstract}

\section{KEYWORDS}

FuZZy Numbers, HEXADECAGONAL NUMBERS, ALPHA CUT, ARITHMETIC OPERATIONS

\section{INTRODUCTION}

L.A.Zadeh introduced fuzzy set theory in1965 [11]. Different types of fuzzy sets are defined in order to clear the vagueness of the existing problems. Membership function of these sets, which have the form $A: R \rightarrow[0,1]$ and it has a quantitative meaning and viewed as fuzzy numbers. Hass. Michael [5], defines a fuzzy number as a quantity whose values are imprecise, rather than exact as in the case with single-valued function. So far, fuzzy numbers like triangular fuzzy numbers [3], trapezoidal fuzzy numbers [1], [10], hexagonal fuzzy numbers [8] are introduced with its membership functions. These numbers have got many applications like non-linear equations, risk analysis and reliability. Many operations were carried out using fuzzy numbers [4]. In this paper, we propose hexadecagonal fuzzy number with its membership functions and also we define basic arithmetic operations of hexadecagonal fuzzy number using arithmetic interval of alpha cuts and is illustrated with numerical examples.

\section{Preliminaries}

\subsection{Fuzzy set [11] :}

A fuzzy set $\bar{A}$ in $\mathrm{X}$ (set of real numbers) is a set of ordered pairs $\bar{A}$ $=\left\{\left(x_{i} \mu_{\bar{A}}(x)\right) / x \in X\right\}_{\mu_{\bar{A}}}(x)$ is called membership function of $\mathrm{x}$ in $\bar{A}$ which maps $\mathrm{X}$ into $[0,1]$.

\subsection{Fuzzy Number [5] :}

A fuzzy set $\bar{A}$ defined on the universal set of real numbers $\mathrm{R}$, is said to be a fuzzy number it its membership function has the following characteristics : 
(i) $\bar{A}$ is convex i.e $\mu_{\bar{A}}\left(\lambda x_{1}+(1-\lambda) x_{2}\right) \geq \min \left(\mu_{\bar{A}}\left(x_{1}\right), \mu_{\bar{A}}\left(x_{2}\right)\right) \forall x_{1}, x_{2} \in R$, $\lambda \in[0,1]$

(ii) $\bar{A}$ is normal i.e., $\exists x_{0} \in R$ such that $\mu_{\bar{A}}\left(x_{0}\right)=1$

(iii) $\mu_{\bar{A}}$ is piecewise continuous.

\subsection{Triangular Fuzzy Number [ 3] :}

A fuzzy number $\bar{A}=(\mathrm{a}, \mathrm{b}, \mathrm{c})$ is said to be a triangular fuzzy number if its membership function is given by,

$$
\mu_{A}(x)=\left\{\begin{array}{cc}
\frac{x-a}{b-a} & \text { for } a \leq x \leq b \\
\frac{c-x}{c b} & \text { for } b \leq x \leq c \\
0 & \text { otherwise }
\end{array}\right.
$$

\subsection{Trapezoidal Fuzzy Number [1] :}

A fuzzy number $\bar{A}=(\mathrm{a}, \mathrm{b}, \mathrm{c}, \mathrm{d})$ is said to be a trapezoidal fuzzy number if its membership function is given by, where $\mathrm{a} \leq \mathrm{b} \leq \mathrm{c} \leq \mathrm{d}$

$$
\mu_{A}(x)=\left\{\begin{array}{cc}
0 & \text { for } x<a \\
\frac{x-a}{b-a} & \text { for } a \leq x \leq b \\
1 & \text { for } b \leq x \leq c \\
\frac{d-x}{d-c} & \text { for } c \leq x \leq d \\
0 & \text { for } x>d
\end{array}\right.
$$

\subsection{Hexagonal Fuzzy Number $[8]$ :}

A fuzzy number $\bar{A}_{H}=\left(a_{1}, a_{2}, a_{3}, a_{4}, a_{5}, a_{6}\right)$ is said to be hexagonal fuzzy number if its membership function is given by

$$
\mu_{\bar{A}_{21}}(x)=\left\{\begin{array}{cl}
0 & \text { for } x \leq a_{1} \\
\frac{1}{2}\left(\frac{x-a_{1}}{a_{2}-a_{1}}\right) & \text { for } a_{1} \leq x \leq a_{2} \\
\frac{1}{2}+\frac{1}{2}\left(\frac{x-a_{2}}{a_{5}-a_{2}}\right) & \text { for } a_{2} \leq x \leq a_{3} \\
1 & \text { for } a_{3} \leq x \leq a_{4} \\
1-\frac{1}{2}\left(\frac{x-a_{4}}{a_{5}-a_{4}}\right) & \text { for } a_{4} \leq x \leq a_{5} \\
\frac{1}{2}\left(\frac{a_{8}-x}{a_{4}-a_{3}}\right) & \text { for } a_{5} \leq x \leq a_{6} \\
0 & \text { for } x>a_{6}
\end{array}\right.
$$

\section{6. $\alpha$-cut of fuzzy set :}

An $\alpha-c u t$ of fuzzy set $\bar{A}$ is a crisp set defined as $A_{\alpha}=\left\{\mathrm{x} \in X / \mu_{\bar{A}}(x) \geq \alpha\right\}$. 


\subsection{Convex fuzzy set:}

A fuzzy set $\bar{A}$ is a convex fuzzy set if and only if each of its $\alpha$-cut $A_{\alpha}$ is a convex set.

\section{HeXadeCagonal Fuzzy Number}

In this section a new form of fuzzy number called Hexadecagonal fuzzy number is introduced which can be much useful in solving many decision making problems.

A fuzzy number $\bar{A}_{H D}=\left(a_{1}, a_{2}, a_{3}, a_{4}, a_{5}, a_{6}, a_{7}, a_{8}, a_{9}, a_{10}, a_{11}, a_{12}, a_{13}, a_{14}, a_{15}, a_{16}\right)$ is said to be Hexadecagonal fuzzy number if its membership function is given by

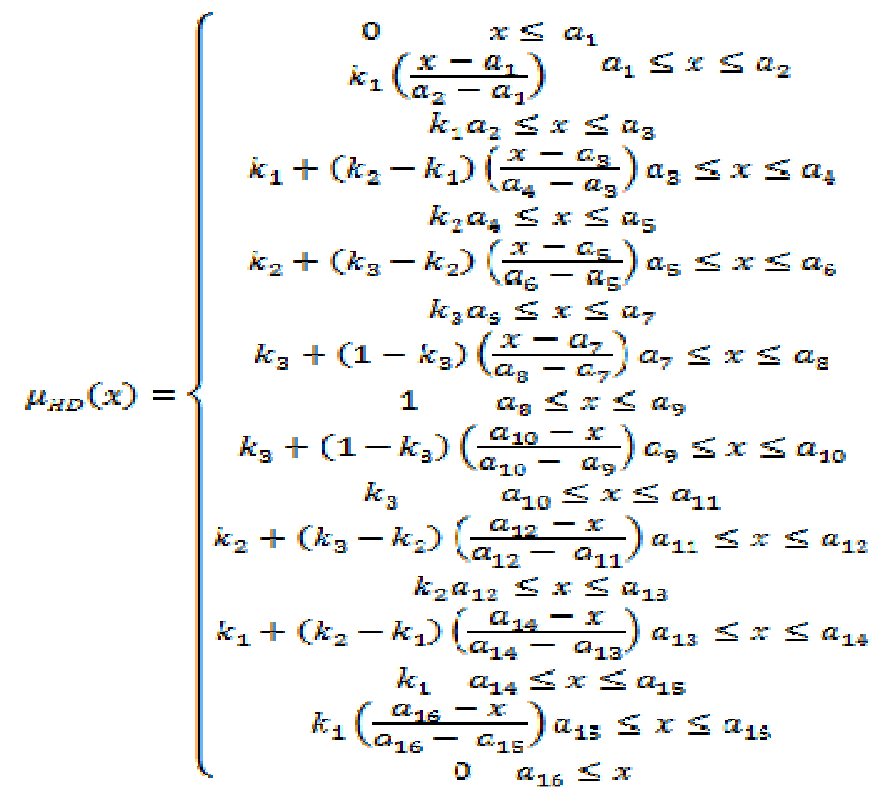

Where $0<k_{1}<k_{2}<k_{3}<1$.

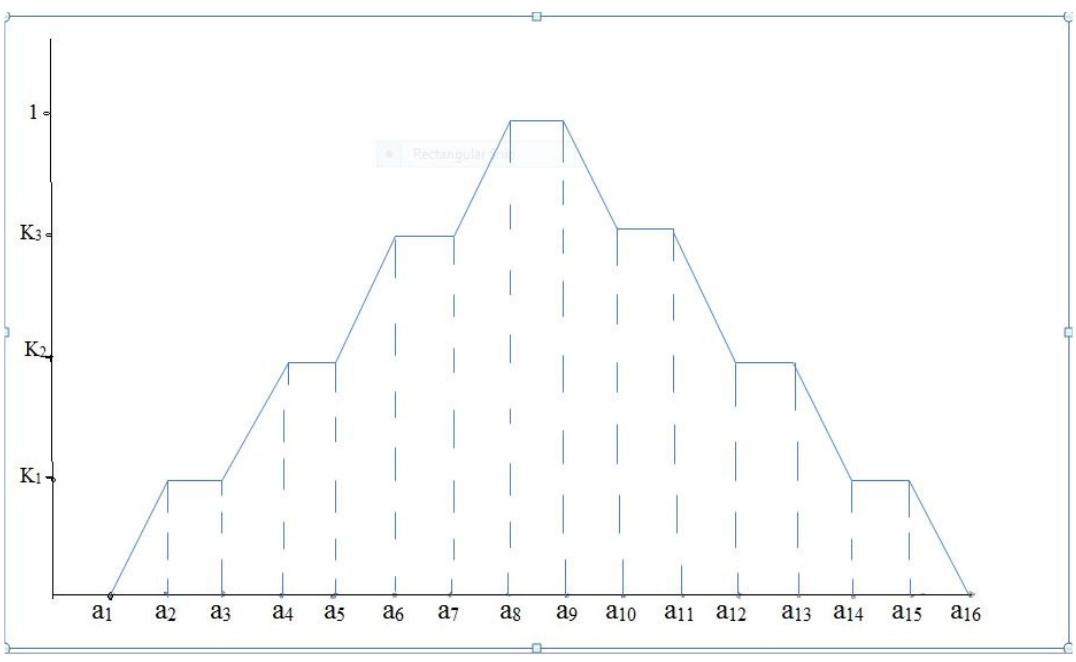

3.1 Graphical representation of Hexadecagonal fuzzy number 


\subsection{Definition:}

The parametric form of Hexadecagonal fuzzy number is defined as

$\bar{A}=\left(f_{1}(p), g_{1}(q), h_{1}(r), l_{1}(s), l_{2}(s), h_{2}(r), g_{2}(q), f_{2}(p)\right)$ for $\mathrm{p} \in\left[0, k_{1}\right], \mathrm{q} \in\left[k_{1}, k_{2}\right]$, $\mathrm{r} \in\left[k_{2}, k_{3}\right] \& \mathrm{~s} \in\left[k_{3}, w\right] . f_{1}(p), g_{1}(q), h_{1}(r), l_{1}(s)$ are bounded left continuous non decreasing functions over $\left[0_{1}, w_{1}\right] \quad\left[k_{1}, w_{2}\right] \quad\left[k_{2}, w_{3}\right]\left[k_{3}, w_{4}\right]$ respectively, $f_{2}(p), g_{2}(q), h_{2}(r), l_{2}(s)$ are bounded left continuous non increasing functions over $\left[0, w_{1}\right]$ $\left[k_{1}, w_{2}\right]\left[k_{2}, w_{3}\right]\left[k_{3}, w_{4}\right]$ respectively, $0 \leq w_{1} \leq k_{1}, k_{1} \leq w_{2} \leq k_{2}, k_{2} \leq w_{3} \leq k_{3}$ and $k_{3} \leq w_{4} \leq w$.

\subsection{Arithmetic Operations on Hexadecagonal Fuzzy Number (HDFN):}

\subsubsection{Addition of two Hexadecagonal Fuzzy Numbers:}

If $\bar{A}_{H D}=\left(a_{1}, a_{2}, a_{3}, a_{4}, a_{5}, a_{6}, a_{7}, a_{8}, a_{9}, a_{10}, a_{11}, a_{12}, a_{13}, a_{14}, a_{15}, a_{16}\right)$

$\bar{B}_{H D}=\left(b_{1}, b_{2}, b_{3}, b_{4}, b_{5}, b_{6}, b_{7}, b_{9}, b_{9}, b_{10}, b_{11}, b_{12}, b_{13}, b_{14}, b_{15}, b_{16}\right)$ then

$\bar{A}_{H D}+\bar{B}_{H D}=\left(a_{1}+b_{1}, a_{2}+b_{2}, a_{3}+b_{3}, a_{4}+b_{4}, a_{5}+b_{5}, a_{6}+b_{6}, a_{7}+b_{7}, a_{8}\right.$

$+b_{8}, a_{9}+b_{9,}, a_{10}+b_{10}, a_{11}+b_{11}, a_{12}+b_{12}, a_{13}+b_{13}, a_{14}+b_{14}, a_{15}$

$\left.+b_{15}, a_{16}+b_{16}\right)$

\section{Example 3.1:}

$$
\begin{gathered}
\text { If } \bar{A}_{H D}=(1,2,3,5,6,8,9,10,11,13,15,16,17,18,19,20) \text { and } \\
\bar{B}_{H D}=(1,3,4,5,6,7,8,9,11,12,13,14,15,16,17,18) \text { then } \\
\bar{A}_{H n}+\bar{B}_{H n}=(2,5,7,10,12,15,17,19,22,25,28,30,32,34,36,38)
\end{gathered}
$$

\subsubsection{Subtraction of two Hexadecagonal Fuzzy Numbers:}

If $\bar{A}_{H D}=\left(a_{1}, a_{2}, a_{3}, a_{4,}, a_{5}, a_{6}, a_{7}, a_{8}, a_{9}, a_{10}, a_{11}, a_{12}, a_{13}, a_{14}, a_{15}, a_{16}\right)$

$\bar{B}_{H D}=\left(b_{1}, b_{2}, b_{3}, b_{4}, b_{5}, b_{6}, b_{7}, b_{3}, b_{9}, b_{10}, b_{11}, b_{12}, b_{13}, b_{14}, b_{15}, b_{16}\right)$ then

$\bar{A}_{H D}-\bar{B}_{H D}=\left(a_{1}-b_{2}, a_{2}-b_{2}, a_{3}-b_{3}, a_{4}-b_{4}, a_{5}-b_{5}, a_{6}-b_{6}, a_{7}-b_{7}, a_{8}\right.$ $-b_{3}, a_{9}-b_{9,}, a_{10}-b_{10}, a_{11}-b_{11}, a_{12}-b_{12}, a_{19}-b_{13}, a_{14}-b_{14}, a_{15}$

Example 3.2:

$$
\left.-b_{15}, a_{16}-b_{16}\right)
$$

$$
\begin{gathered}
\text { If } \bar{A}_{H D}=(1,3,7,9,12,14,16,18,20,23,25,27,29,31,33,36) \text { and } \\
\bar{B}_{H D}=(0,1,2,3,4,5,6,7,8,10,11,12,13,14,15,16) \text { then } \\
\bar{A}_{H D}-\bar{B}_{H D}=(1,2,5,6,8,9,10,11,12,13,14,15,16,17,18,20)
\end{gathered}
$$

\subsubsection{Scalar Multiplication of two Hexadecagonal Fuzzy Numbers:}

If $\bar{A}_{H D}=\left(a_{1}, a_{2}, a_{3}, a_{4}, a_{5}, a_{6}, a_{7}, a_{8}, a_{9}, a_{10}, a_{11}, a_{12}, a_{13}, a_{14}, a_{15}, a_{16}\right)$

Then $k A_{H D}=$

$\left(k a_{1}, k a_{2}, k a_{3}, k a_{4}, k a_{5}, k a_{6}, k a_{7}, k a_{8}, k a_{9}, k a_{10}, k a_{11}, k a_{12}, k a_{13}, a_{14}, a_{15}, a_{16}\right)$ 


\section{Example 3.3}

$$
\text { If } \begin{aligned}
\bar{A}_{H D} & =(1,2,3,5,6,8,9,10,11,13,15,16,17,18,19,20) \\
2 \bar{A}_{H D} & =(2,4,6,10,12,16,18,20,22,26,30,32,34,36,38,40)
\end{aligned}
$$

\subsubsection{Multiplication of two Hexadecagonal Fuzzy Numbers:}

If $\bar{A}_{H D}=\left(a_{1}, a_{2}, a_{3}, a_{4,}, a_{5}, a_{6,}, a_{7}, a_{8}, a_{9,} a_{10}, a_{21}, a_{12}, a_{13}, a_{14}, a_{25}, a_{26}\right)$ $\bar{B}_{H D}=\left(b_{1}, b_{2}, b_{3}, b_{4}, b_{5}, b_{6}, b_{7}, b_{3}, b_{9}, b_{10}, b_{11}, b_{12}, b_{13}, b_{14}, b_{15}, b_{16}\right)$ then

$$
\begin{aligned}
\bar{A}_{H D} * \bar{B}_{H D}= & \left(a_{1} * b_{1}, a_{2} * b_{2}, a_{3} * b_{3}, a_{4} * b_{4}, a_{5} * b_{5}, a_{6} * b_{6}, a_{7} * b_{7}, a_{8} * b_{8}, a_{9}\right. \\
& * b_{9}, a_{10} * b_{10}, a_{11} * b_{11}, a_{12} * b_{12}, a_{13} * b_{13}, a_{14} * b_{14}, a_{15} * b_{15}, a_{16} \\
& \left.* b_{16}\right)
\end{aligned}
$$

\section{Example 3.4:}

If $\bar{A}_{H D}=(0,1,2,3,4,5,6,7,8,9,10,11,12,13,14,15)$ and

$\bar{B}_{H D}=(1,2,3,4,5,6,7,8,9,10,11,12,13,14,15,16)$ then

$\bar{A}_{H D} * \bar{B}_{H D}=(0,2,6,12,20,30,42,56,72,90,110,132,156,182,210,240)$

\subsubsection{Equal Hexadecagonal Fuzzy Numbers :}

Two Hexadecagonal Fuzzy Numbers

$\bar{A}_{H D}=\left(a_{1}, a_{2}, a_{3}, a_{4}, a_{5}, a_{6}, a_{7}, a_{9}, a_{9,}, a_{10}, a_{11}, a_{12}, a_{13}, a_{14}, a_{15}, a_{16}\right)$

$\bar{B}_{H D}=\left(b_{1}, b_{2}, b_{3}, b_{4}, b_{5}, b_{6}, b_{7}, b_{8}, b_{9}, b_{10}, b_{11}, b_{12}, b_{13}, b_{14}, b_{15}, b_{16}\right)$ are equal i.e. $\bar{A}_{H D}=\bar{B}_{H D}$ iff $a_{i}=b_{i} \forall i$

\subsubsection{Positive Hexadecagonal Fuzzy Number:}

A positive Hexadecagonal Fuzzy Number (p-HDFN) is defined as $p-\bar{A}_{H D}=\left(a_{1}, a_{2}, a_{3}, a_{4}, a_{5}, a_{6}, a_{7}, a_{8}, a_{9}, a_{10}, a_{11}, a_{12}, a_{13}, a_{14}, a_{15}, a_{16}\right)$ where $a_{i}>0$ Y $i$.

\section{Example 3.5:}

p- $\bar{A}_{H D}=(1,2,3,5,6,8,9,10,11,13,15,16,17,18,19,20)$

\subsubsection{Negative Hexadecagonal Fuzzy Number:}

A negative Hexadecagonal Fuzzy Number (n-HDFN) is defined as $n-\bar{A}_{H n}=\left(a_{1}, a_{2}, a_{3}, a_{4}, a_{5}, a_{6}, a_{7}, a_{8}, a_{9,}, a_{10}, a_{11}, a_{12}, a_{13}, a_{14}, a_{15}, a_{16}\right)$ where $a_{i} \leqslant 0 \forall i$

\section{Example 3.6:}

$$
n-\bar{A}_{H D}=(-20,-19,-18,-17,-16,-15,-13,-11,-10,-9,-8,-6,-5,-3,-2,-1)
$$




\section{Alpha Cut}

\subsection{Definition:}

For $\alpha \in[0,1]$, the $\alpha-$ cut of Hexadecagonal fuzzy number ,

$A_{H D}=\left(a_{1}, a_{2}, a_{3}, a_{4}, a_{5}, a_{6}, a_{7}, a_{8}, a_{9,}, a_{10}, a_{11}, a_{12}, a_{13}, a_{14}, a_{15}, a_{16}\right)$ is defined as $\left[\bar{A}_{H D}\right]_{\alpha}=$

$\left[\alpha_{1}+\frac{\alpha}{k_{1}}\left(\alpha_{2}-\alpha_{1}\right), \quad \alpha_{16}-\frac{\alpha}{k_{1}}\left(\alpha_{16}-\alpha_{16}\right)\right]$ for $\alpha \in\left[0, k_{1}\right]$

$\left[a_{3}+\left(\frac{\alpha-k_{1}}{k_{2}-k_{1}}\right)\left(a_{4}-a_{3}\right), a_{14}-\left(\frac{\alpha-k_{1}}{k_{2}-k_{1}}\right)\left(a_{14}-a_{13}\right)\right]$ for $\alpha \in\left\lceil k_{1}, k_{2}\right\rceil$

$\left[a_{5}+\left(\frac{\alpha-k_{2}}{k_{5}-k_{2}}\right)\left(a_{6}-a_{5}\right), a_{12}-\left(\frac{\alpha-k_{2}}{k_{5}-k_{2}}\right)\left(a_{12}-a_{11}\right)\right]$ for $a \in\left[k_{2}, k_{3}\right]$

$\left[a_{7}+\left(\frac{\alpha-k_{\mathrm{g}}}{1-k_{\mathrm{g}}}\right)\left(a_{0}-a_{7}\right), a_{10}-\left(\frac{\alpha-k_{\mathrm{g}}}{1-k_{\mathrm{g}}}\right)\left(a_{10}-a_{0}\right)\right]$ for $\alpha \in\left[k_{3}, 1\right]$

\subsection{Operations of hexadecagonal fuzzy numbers using $\propto$ - Cut:}

The $\propto$ - Cut of hexadecagonal fuzzy number

$\bar{A}_{H D}=\left(a_{1}, a_{2}, a_{3}, a_{4}, a_{5}, a_{6}, a_{7}, a_{3}, a_{9}, a_{10}, a_{11}, a_{12}, a_{13}, a_{14}, a_{15}, a_{16}\right)$ for all $\kappa \in[0,1]$ when $k_{1}=\frac{1}{4}, k_{2}=\frac{1}{2}, k_{3}=\frac{3}{4}$ is given by

$$
\begin{aligned}
& {\left[\bar{A}_{H D}\right]_{\alpha}} \\
& \left\{\begin{array}{cc}
{\left[a_{1}+4 \propto\left(a_{2}-a_{1}\right), a_{16}-4 \propto\left(a_{16}-a_{15}\right)\right]} & \text { for } \propto \in[0.0 .25] \\
{\left[a_{3}+(4 \alpha-1)\left(a_{4}-a_{3}\right), a_{14}-(4 \alpha-1)\left(a_{14}-a_{13}\right)\right]} & \text { for } \alpha \in[0.25 .0 .5] \\
{\left[a_{5}+(4 \propto-7)\left(a_{6}-a_{5}\right), a_{12}-(4 \alpha-7)\left(a_{12}-a_{11}\right)\right]} & \text { for } \propto f[0.5,0.75] \\
{\left[a_{3}+(4 \alpha-3)\left(a_{8}-a_{7}\right), a_{10}-(4 \alpha-3)\left(a_{10}-a_{4}\right)\right]} & \text { for } \propto \in[0.75,1]
\end{array}\right.
\end{aligned}
$$

\subsubsection{Addition:}

Let $\bar{A}_{H D}=\left(a_{1}, a_{2}, a_{3}, a_{4}, a_{5}, a_{6}, a_{7}, a_{8}, a_{9}, a_{10}, a_{11}, a_{12}, a_{13}, a_{14}, a_{16}, a_{16}\right)$

$\bar{B}_{H D}=\left(b_{1}, b_{2}, b_{3}, b_{4}, b_{5}, b_{6}, b_{7}, b_{8}, b_{9}, b_{10}, b_{11}, b_{12}, b_{13}, b_{14}, b_{15}, b_{16}\right)$

be two hexadecagonal fuzzy numbers. Let us add the alpha cuts of $\left[\bar{A}_{H D}\right]_{\omega}$ and $\left[\bar{F}_{H D}\right]_{\alpha}$ of $\bar{A}_{H D}$ and $\bar{B}_{H E}$ using interval arithmetic.

$\left[\bar{A}_{H D}\right]_{\alpha}+\left[\bar{B}_{H D}\right]_{\alpha}=$

$\left\{\begin{array}{c}{\left[\begin{array}{c}{\left[a_{1}+4 \propto\left(a_{2}-a_{1}\right), a_{16}-4 \propto\left(a_{16}-a_{15}\right)\right]+} \\ {\left[b_{1}+4 \propto\left(b_{2}-b_{1}\right), b_{16}-4 \propto\left(b_{16}-b_{15}\right)\right]}\end{array}\right] \text { for } \propto \in[0,0.25]} \\ {\left[\begin{array}{c}{\left[a_{3}+(4 \alpha-1)\left(a_{4}-a_{3}\right), a_{14}-(4 \alpha-1)\left(a_{14}-a_{13}\right)\right]+} \\ {\left[b_{3}+(4 \alpha-1)\left(b_{4}-b_{3}\right), b_{14}-(4 \alpha-1)\left(b_{14}-b_{13}\right)\right]}\end{array}\right] \text { for } \propto \in[0.25,0.5]} \\ {\left[\begin{array}{cc}{\left[a_{5}+(4 \alpha-2)\left(a_{6}-a_{5}\right), a_{12}-(4 \alpha-2)\left(a_{12}-a_{11}\right)\right]+} \\ {\left[b_{5}+(4 \alpha-2)\left(b_{6}-b_{5}\right), b_{12}-(4 \alpha-2)\left(b_{12}-b_{11}\right)\right]}\end{array}\right] \text { for } \alpha \in[0.5,0.75]} \\ {\left[\begin{array}{c}{\left[a_{7}+(4 \alpha-3)\left(a_{8}-a_{7}\right), a_{10}-(4 \alpha-3)\left(a_{10}-a_{9}\right)\right]+} \\ {\left[b_{7}+(4 \alpha-3)\left(b_{8}-b_{7}\right), b_{10}-(4 \alpha-3)\left(b_{10}-b_{9}\right)\right]}\end{array}\right] \text { for } \propto \in[0.75,1]}\end{array}\right.$ 


\section{Example 4.1:}

If $\bar{A}_{H_{D}}=(1,2,3,5,6,8,9,10,11,13,15,16,17,18,19,20)$ and

$\bar{B}_{H D}=(1,3,4,5,6,7,8,9,11,12,13,14,15,16,17,18)$

For $\propto \in[0,0.25]\left[\bar{A}_{H D}\right]_{\alpha}=[1+4 \propto, 20-4 \propto]\left[\bar{B}_{H D}\right]_{\alpha}=[1+8 \propto, 19-4 \propto]$

$\left[\bar{A}_{H n}\right]_{\sigma x}+\left[\bar{B}_{\mu n}\right]_{\kappa}=[2+12 \propto, 38-8 \times]$

When $\propto=0 \quad\left[\bar{A}_{H D}\right]_{0}+\left[\bar{B}_{H D}\right]_{0}=[2,38]$

When $\propto=0.25\left[\bar{A}_{H D}\right]_{0.25}+\left[\bar{B}_{H D}\right]_{0.25}=[5,36]$

For $\propto \in[0.25,0.5]\left[\bar{A}_{H D}\right]_{\alpha}-[1+8 \kappa, 19-4 \propto]\left[\bar{b}_{H D}\right]_{\alpha}-[3+4 \kappa, 17-4 \kappa]$

$\left[\bar{A}_{H D}\right]_{\alpha}+\left[\bar{B}_{H D}\right]_{\alpha}=[4+12 \propto, 36-8 \times]$

When $\propto=0.25\left[\bar{A}_{H D}\right]_{0.2 \mathrm{~s}}+\left[\bar{B}_{H D}\right]_{0.2 \mathrm{~s}}=[7,34]$

When $\propto=0.5\left[\bar{A}_{H D}\right]_{0.5}+\left[\bar{B}_{H D}\right]_{0.5}=[10,32]$

For $\propto \in[0.5,0.75] \quad\left[\bar{A}_{H D}\right]_{\alpha}=[2+8 \propto, 18-4 \propto]\left[\bar{B}_{H D}\right]_{\alpha}=[4+4 \propto, 16-4 \propto]$

$\left[\bar{A}_{H D}\right]_{\alpha} \|\left[\bar{B}_{H D}\right]_{\alpha}=[6+12 \propto, 34-8 \times]$

When $\alpha=0.5\left[\bar{A}_{H D}\right]_{0.5}+\left[\bar{B}_{H D}\right]_{0.5}=[12,30]$

When $\alpha=0.75\left[\bar{A}_{\text {us }}\right\rceil_{0.75}+\left[\bar{B}_{\text {HD }}\right\rceil_{075}=[15,28]$

For $\propto \in[0.75,1] \quad\left[\bar{A}_{H D}\right]_{\alpha}=[6+4 \propto, 19-8 \propto] \quad\left[\bar{B}_{H D}\right]_{\alpha}=[5+4 \propto, 15-4 \propto]$

$\left[\bar{A}_{H D}\right]_{\alpha}+\left[\bar{B}_{H D}\right]_{\alpha}=[11+8 \propto, 34-12 \propto]$

When $\propto=0.75\left[\bar{A}_{H D}\right]_{0.75}+\left[B_{H D}\right]_{0.75}=[17,25]$

When $\propto=1\left[\bar{A}_{H D}\right]_{1}+\left[\bar{B}_{H D}\right]_{1}=[19,22]$

Hence $\bar{A}_{H n}+\bar{B}_{H n}=(2,5,7,10,12,15,17,19,22,25,28,30,32,34,36,38)$

\subsubsection{Subtraction:}

Let $\bar{A}_{H D}=\left(a_{1}, a_{2}, a_{3}, a_{4}, a_{5}, a_{6}, a_{7}, a_{8}, a_{9}, a_{10}, a_{11}, a_{12}, a_{13}, a_{14}, a_{15}, a_{16}\right)$

$\bar{D}_{H D}=\left(b_{1}, b_{2}, b_{3}, b_{4}, b_{5}, b_{6}, b_{7}, b_{3}, b_{9}, b_{10}, b_{11}, b_{12}, b_{13}, b_{14}, b_{15}, b_{16}\right)$

be two hexadecagonal fuzzy numbers . Let us subtract the alpha cuts of $\left[\bar{A}_{H D}\right]_{u}$ and $\left[\bar{R}_{H D}\right]_{u}$ of $\bar{A}_{H D}$ and $\bar{B}_{H D}$ using interval arithmetic.

$$
\begin{aligned}
& {\left[\bar{A}_{H D}\right]_{\alpha}-\left[\bar{B}_{H D}\right]_{\alpha}=}
\end{aligned}
$$

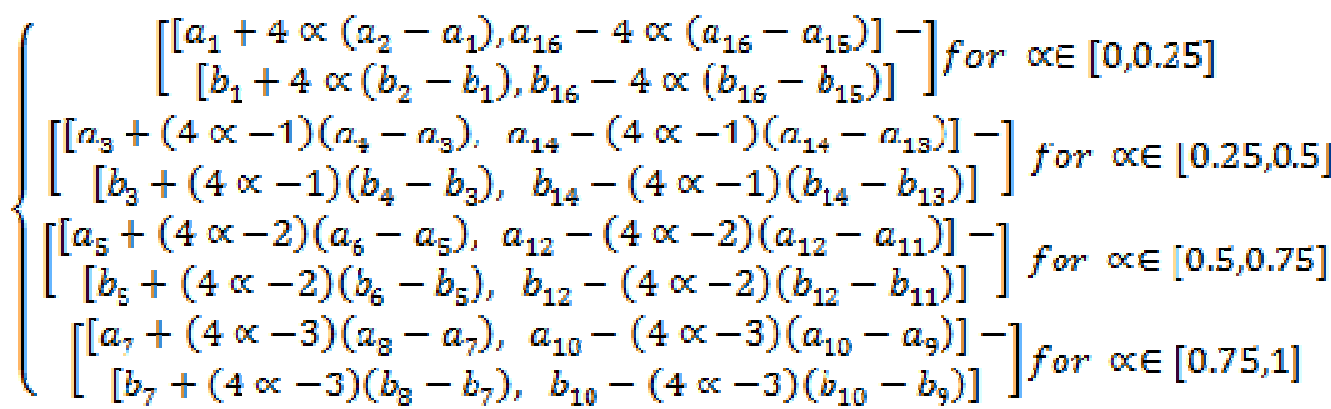

\section{Example 4.2}

$$
\text { If } \begin{aligned}
\bar{A}_{H D} & =(1,3,7,9,12,14,16,18,20,23,25,27,29,31,33,36) \text { and } \\
\bar{B}_{H D} & =(0,1,2,3,4,5,6,7,8,10,11,12,13,14,15,16)
\end{aligned}
$$

For $\propto \in[0,0.25]\left[\bar{A}_{H D}\right]_{\alpha}=[1+8 \propto, 36-12 \propto]\left[\bar{B}_{H D}\right]_{\alpha}=[4 \propto, 16-4 \propto]$ $\left\lfloor A_{H D}\right\rfloor_{\alpha}-\left\lfloor B_{H D}\right\rfloor_{\alpha}=[1+4 \propto, 20-8 \propto]$ 
When $\propto=0 \quad\left[\bar{A}_{H D}\right]_{D}-\left[\bar{B}_{H D}\right]_{0}=[1,20]$

When $\propto=0.25\left[\bar{A}_{H D}\right]_{0.25}-\left[\bar{B}_{H D}\right]_{0.25}=[2,18]$

For $\propto \in[0.25,0.5]\left[\bar{A}_{H D}\right]_{\alpha}=[5+8 \propto, 33-8 \propto]\left[\bar{B}_{H D}\right]_{\alpha}=[1+4 \propto, 15-4 \propto]$

$\left\lceil\bar{A}_{H D}\right]_{\alpha}-\left[\bar{B}_{H D}\right]_{\alpha}=[4+4 \propto, 18-4 \alpha]$

When $\propto=0.25\left[\bar{A}_{H D}\right]_{0.25}-\left[\bar{B}_{H D}\right]_{0.25}=[5,17]$

When $\propto=0.5\left[\bar{A}_{H n}\right]_{\mathrm{QS}}-\left[\bar{B}_{H n}\right]_{\mathrm{n} .5}=[6,16]$

For $\propto \in[0.5,0.75] \quad\left[\bar{A}_{H D}\right]_{\alpha}=[8+8 \propto, 31-8 \propto]\left[\bar{B}_{H D}\right]_{\alpha}=[2+4 \propto, 14-4 \propto]$

$\left[\bar{A}_{H D}\right]_{\alpha}-\left[\bar{B}_{H D}\right]_{\alpha}=[6+4 \propto, 17-4 \propto]$

When $\propto=0.5\left[\bar{A}_{\mu n}\right]_{\Omega, 5}-\left[\bar{B}_{\mu n}\right]_{\Omega:=}=[8,15]$

When $\propto=0.75\left[\bar{A}_{H D}\right]_{0.7 \mathrm{~s}}-\left[\bar{B}_{H D}\right]_{0.7 \mathrm{~s}}=[9,14]$

For $\propto \in[0.75,1]\left[\bar{A}_{H D}\right]_{\alpha}=[10+B \propto, 32-12 \propto]\left[\bar{B}_{H D}\right]_{\alpha}=[3+4 \propto, 16-8 \propto]$

$\left[\bar{A}_{H D}\right]_{\alpha}-\left[\bar{B}_{H D}\right]_{\alpha}-[7+4 \propto, 16-4 \propto]$

When $\alpha=0.75\left[\bar{A}_{H D}\right]_{0.7 \mathrm{~s}}-\left[\bar{B}_{H D}\right]_{0.7 \mathrm{~s}}=[10,13]$

When $\propto=1\left[\bar{A}_{H D}\right]_{1}-\left[\bar{A}_{H D}\right]_{1}=[11,12]$

Hence $\bar{A}_{H D}-\bar{B}_{H D}=(1,2,5,6,8,9,10,11,12,13,14,15,16,17,18,20)$

\subsubsection{Scalar Multiplication:}

Let $A_{H D}=\left(a_{1}, a_{2}, a_{3}, a_{4,}, a_{5}, a_{6}, a_{7}, a_{8}, a_{9}, a_{10}, a_{11}, a_{12}, a_{13}, a_{14}, a_{15}, a_{16}\right)$

be a hexadecagonal fuzzy number. Let us find the scalar multiplication of alpha cuts $\left[\bar{A}_{H D}\right]_{\alpha}$ of $\bar{A}_{H D}$ using interval arithmetic.

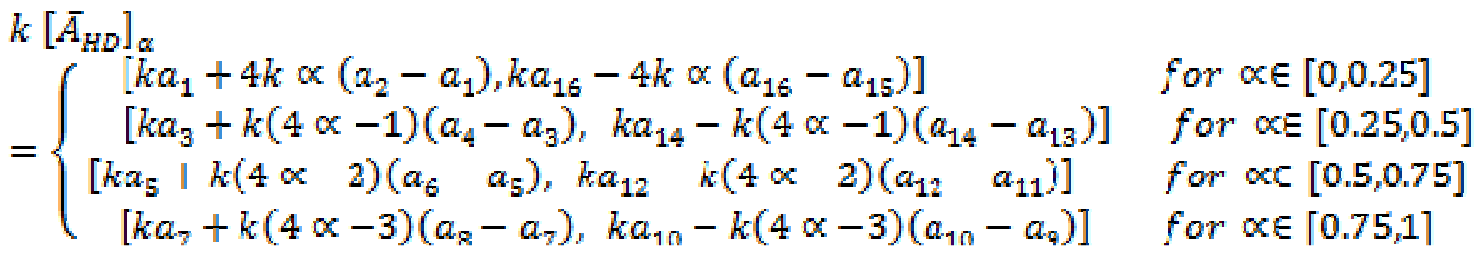

\section{Example 4.3:}

If $\bar{A}_{H D}=(1,2,3,5,6,8,9,10,11,13,15,16,17,18,19,20)$

For $\propto \in[0,0.25] \quad\left[\bar{A}_{H D}\right]_{\alpha}=[1+4 \propto, 20-4 \propto] \quad 2\left[\bar{A}_{H D}\right]_{\alpha}=[2+8 \propto, 40-8 \propto]$

$2\left[\bar{A}_{H D}\right]_{0}=[2,40] 2\left[\bar{A}_{H D}\right]_{0.25}=[4,38]$

For $\propto \in[0.25,0.5] \quad\left[\bar{A}_{H D}\right]_{\alpha}=[1+8 \propto, 19-4 \propto] \quad 2\left[\bar{A}_{H D}\right]_{\alpha}=[2+16 \propto, 38-8 \propto]$

$2\left[\bar{A}_{H D}\right]_{0.25}=\lceil 6,36\rceil 2\left\lceil\bar{A}_{H \Omega}\right]_{0.5}=\lceil 10,34\rceil$

For $\propto \in[0.5,0.75]\left[\bar{A}_{H D}\right]_{\alpha}=[2+8 \propto, 18-4 \propto] \quad 2\left[\bar{A}_{H D}\right]_{\alpha}=[4+16 \propto, 36-8 \propto]$

$2\left[\bar{A}_{H N}\right]_{\mathrm{RS}}=[12,32] 2\left[\bar{A}_{H N}\right]_{\mathrm{O} . \mathrm{FE}}=[16,30]$

For $\propto \in[0.75,1] \quad\left[\bar{A}_{H D}\right]_{\alpha}=[6+4 \propto, 19-8 \alpha] 2 \quad\left[\bar{A}_{H D}\right]_{\alpha}=[12+8 \propto, 38-16 \alpha]$

$2\left[\bar{A}_{H D}\right]_{0.75}=[18,26] 2\left[\bar{A}_{H D}\right]_{1}=[20,22]$

Hence $2 \bar{A}_{H L}=(2,4,6,10,12,16,18,20,22,26,30,32,34,36,38,40)$

\subsubsection{Multiplication:}

Let $\bar{A}_{H N}=\left(a_{1}, a_{2}, a_{3}, a_{4}, a_{5}, a_{6,} a_{7}, a_{8}, a_{9}, a_{10}, a_{11}, a_{12}, a_{13}, a_{14}, a_{15}, a_{16}\right)$

$\bar{B}_{H D}=\left(b_{1}, b_{2}, b_{3}, b_{4}, b_{5}, b_{6}, b_{7}, b_{9}, b_{9}, b_{10}, b_{11}, b_{12}, b_{13}, b_{14}, b_{15}, b_{16}\right)$

be two hexadecagonal fuzzy numbers. Let us multiply the alpha cuts of $\left[\bar{A}_{H D}\right]_{x}$ and $\left[\bar{B}_{H D}\right]_{\alpha}$ of $\bar{A}_{I L D}$ and $\bar{B}_{l l D}$ using interval arithmetic. 


$$
\begin{aligned}
& {\left[\bar{A}_{H D}\right]_{\alpha} *\left[\bar{B}_{M D}\right]_{\alpha}=}
\end{aligned}
$$

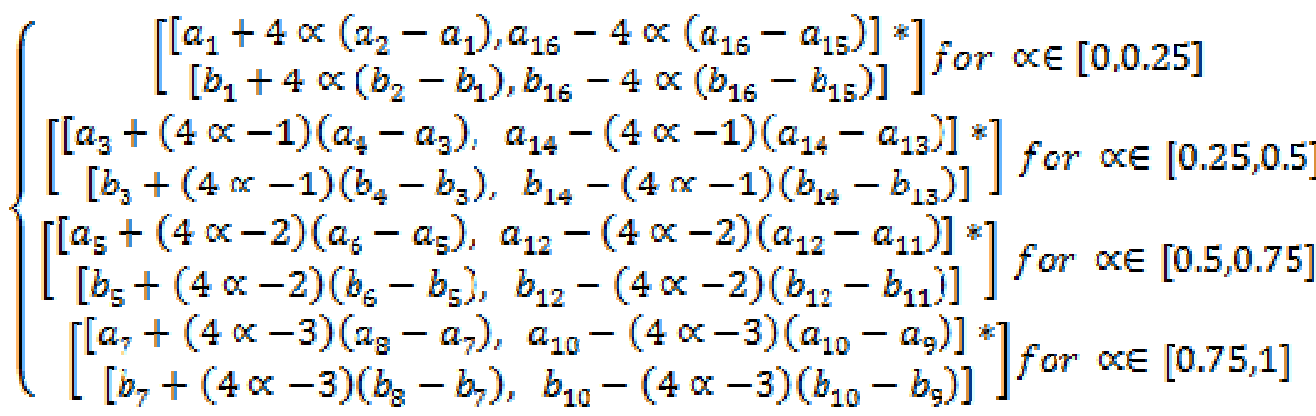

\section{Example 4.4:}

If $\overline{\boldsymbol{A}}_{H D}=(0,1,2,3,4,5,6,7,8,9,10,11,12,13,14,15)$ and

$\bar{B}_{H D}=(1,2,3,4,5,6,7,8,9,10,11,12,13,14,15,16)$

For $\propto \in[0,0.25] \quad\left[\bar{A}_{H N}\right]_{n}=[4 \propto, 15-4 \propto] \quad\left[\bar{B}_{H N}\right]_{n}=[1+4 \propto, 16-4 \propto]$

$\left[\bar{A}_{H D}\right]_{\alpha} *\left[\bar{B}_{H D}\right]_{\alpha}=[4 \alpha(1+4 \alpha),(15-4 \alpha)(16-\alpha)]$

$\propto=0 \quad\left[\bar{A}_{H D}\right]_{0} *\left[\bar{B}_{H D}\right]_{0}=[0,240]$

$\propto=0.25\left[\bar{A}_{H D}\right]_{0.2 \mathrm{E}} *\left[\bar{B}_{H D}\right]_{0.2 \mathrm{E}}=[2,210]$

For $\propto \in[0.25,0.5]\left[\bar{A}_{H D}\right]_{\alpha}=[1+4 \propto, 14-4 \propto]\left[\bar{B}_{H D}\right]_{\alpha}=[2+4 \propto, 15-4 \propto]$

$\left[\bar{A}_{H D}\right]_{\alpha} *\left[\bar{B}_{H N}\right]_{\alpha}=[(1+4 \propto)(2+4 \propto),(14-4 \propto)(15-4 \propto)]$

When $\alpha=0.25\left[\bar{A}_{H D}\right]_{0.25} *\left[B_{H D}\right]_{0.2 \mathrm{E}}=[6,182]$

When $\propto=0.5\left[\bar{A}_{H D}\right]_{115} *\left[\bar{B}_{H D}\right]_{0.5}=[12,156]$

For $\propto[0.5,0.75]\left[\bar{A}_{H D}\right]_{\alpha}-[2+4 \propto, 13-4 \propto]\left[\bar{B}_{H D}\right]_{\alpha}-[3+4 \propto, 14-4 \propto]$

$\left[\bar{A}_{H D}\right]_{\alpha} *\left[\bar{B}_{H D}\right]_{\alpha}=\left[(2+4 \propto)(3+4 \alpha)_{1}(13-4 \propto)(14-4 \propto)\right]$

When $\alpha=0.5\left[\bar{A}_{H D}\right]_{\text {iD. }} *\left[\bar{B}_{H D}\right]_{0.5}=[20,132]$

When $\mathrm{x}=0.75\left[\bar{A}_{H D}\right]_{0.75} *\left[\bar{B}_{H D}\right]_{0.75}=[30,110]$

For $\propto \in[0.75,1]\left[\bar{A}_{H D}\right]_{\alpha}=[3+4 \propto, 12-4 \propto]\left[\bar{B}_{H D}\right]_{\alpha}=[4+4 \propto, 13-4 \propto]$

$\left[\bar{A}_{H D}\right]_{\alpha} *\left[\bar{B}_{H D}\right]_{\alpha}=[(3+4 \alpha)(4+4 \alpha)(12-4 \alpha)(13-4 \alpha)]$

When $\propto=0.75\left[\bar{A}_{H D}\right]_{0.75} *\left[\bar{B}_{H D}\right]_{0.75}=[42,90]$

When $\propto=1\left[\bar{A}_{H D}\right]_{1} *\left[\bar{B}_{H D}\right]_{1}=[56,72]$

Hence $\bar{A}_{H D} * \bar{B}_{H D}=(0,2,6,12,20,30,42,56,72,90,110,132,156,182,210,240)$

\section{Conclusion}

In this paper, a new form of fuzzy number named as Hexa-decagonal Fuzzy Number is introduced. The arithmetic operations are performed with arithmetic interval of alpha cuts and are illustrated with numerical examples. Hexa-decagonal Fuzzy Number can be applied to that problem which has sixteen points in representation. In future, it may be applied in operations research problems.

\section{REFERENCES}

[1] Bansal. Abhinav., (2011), Trapezoidal Fuzzy Numbers (a,b,c,d); Arithmetic Behaviour, International Journal of Physical and Mathematical Sciences, ISSN: 2010-1791.

[2] Bansal,A.,(2010)Some nonlinear arithmetic operations on triangular fuzzy numbers (m,B, a) . Advances in fuzzy mathematics, 5,147-156. 
[3] Dinagar. D. Stephen and Latha. K., (2013),Some types of Type-2 Triangular FuzzyMatrices, International Journal of Pure and Applied Mathematics, Vol-82, No.1, 21-32.

[4] Dubois. D and Prade. H., Operations on Fuzzy Numbers, International Journal of Systems Science, Vol-9, No.6., pp.613-626.

[5] Hass. Michael., (2009), Applied Fuzzy Arithmetic, Springer International Edition,ISBN 978-81-8489300.

[6] Kauffmann,A.,(1980) Gupta,M., Introduction to Fuzzy Arithmetic :Theory and Applications, VanNostrand Reinhold, New York.

[7] Klir. G.J and Bo Yuan., (2005), Fuzzy Sets and Fuzzy logic, Prentice Hall of India Private Limited.

[8] Rajarajeswari.P and Sahaya Sudha.A., (2014), A New Approach for Ranking of Fuzzy Numbers using the Incentre of Centroids, International Journal of Fuzzy Mathematical Archive,Vol-4,52-60.

[9] Parvathi.C and Malathi.C., (2012), Arithmetic operations on Symmetric Trapezoidal Intuitionistic Fuzzy Numbers, International Journal of Soft Computing and Engineering, ISSN: 2231-2307, Vol-2.

[10] Rezvani .S.,(2011), Multiplication Operation on Trapezoidal Fuzzy numbers, Journal of Physical Sciences, Vol no-15,17-26

[11] Zadeh,L.A.,(1978) , Fuzzy set as a basis for a theory of possibility, Fuzzy sets and systems, No. 1, pp.3-28.

\section{Authors}

Dr. A. Sahaya Sudha, Assistant Professor, Department of Mathematics, Nirmala College for women, Coimbatore. She is in the field of Research and Teaching for 19 years. She has published more than 23 papers in various prestigious international journals with high impact factor. She has produced 10 M.Phil. Research scholars with high credibility. Her area of interest includes Operation Research.

R. Gokilamani, Associate Professor, Department of Mathematics, Sri Ramakrishna College of Arts and Science for Women, Coimbatore. She is in the field of Teaching for 18 years. Her area of specialisation is Operations Research. 